\title{
LOS SISTEMAS DE EVALUACIÓN DE TRABAJADORES BASADOS EN LAS VALORACIONES DE LOS CLIENTES
}

\author{
Albert Bach Gibert \\ Abogado de Cuatrecasas
}

\begin{abstract}
Los sistemas de evaluación del individuo por parte de la sociedad han pasado de ser ciencia ficción a convertirse en una realidad en países como China. La utilización de este tipo de modelos en la evaluación de los trabajadores ya es posible en varias plataformas digitales y es cuestión de tiempo que evaluar a un trabajador se convierta en algo tan cotidiano como evaluar a un restaurante o a un posible pretendiente en una aplicación de citas. En este artículo pretendemos acercar al lector las problemáticas jurídicas que pueden surgir del uso de dichos sistemas en el ámbito del Derecho Laboral, en particular desde la óptica del derecho a la igualdad y a la no discriminación.

Evaluation systems in which society rates individuals were envisioned as science-fiction but they have become a reality in countries like China. The use of these systems to evaluate employees is possible in several digital platforms and it is only a matter of time that rating a worker becomes as ordinary as rating a restaurant or a possible match in a dates app. This article approaches the reader with the legal issues that may arise from the use of these systems from a Labour Law perspective, in particular from an equality and non-discrimination rights point of view.
\end{abstract}

Title: The employees evaluation systems based on the consumer's ratings.

Palabras clave: evaluación, trabajadores, discriminación, plataformas digitales, tecnología.

Keywords: evaluation, employees, discrimination, digital platforms, technology.

IUSLabor 3/2019, ISSN 1699-2938, p. 6-30

DOI. 10.31009/IUSLabor.2019.i03.01

Fecha envío: 1.10.2019 | Fecha aceptación: 12.11.2019 


\section{Sumario}

1. Introducción

2. Los sistemas de evaluación

2.1. ¿Por qué existen y en qué consisten estos sistemas de evaluación?

2.2. ¿Qué empresas usan este sistema?

2.3. ¿Existen sesgos en las evaluaciones de los clientes?

3. ¿Cómo afectan estos sistemas al derecho a la igualdad y a la no discriminación?

3.1. ¿Existe una vulneración del derecho a la igualdad y a la no discriminación?

3.2. ¿Sería jurídicamente viable una reclamación por discriminación?

4. Posibles modificaciones en el sistema de evaluación

5. Conclusión

5. Bibliografía 


\section{Introducción}

En el primer episodio de la tercera temporada de la conocida serie Black Mirror se presentó al público una ficción terriblemente realista de un mundo en el que las personas tenían asignada una reputación digital que incrementaba o disminuía por las valoraciones de sus conciudadanos y del Estado. Dicha reputación tenía consecuencias en cuanto al precio del alquiler, a la accesibilidad del ciudadano a determinados servicios y finalmente tenía consecuencias respecto de su trabajo.

En el presente artículo queremos acercar al lector las problemáticas jurídicas desde un punto de vista laboral de los sistemas de evaluación de trabajadores que, basándose en un sistema similar al que se mostraba en el citado episodio, establecen una puntuación del trabajador que depende directamente de las valoraciones directas de los clientes.

Si bien este sistema de evaluación no es muy conocido actualmente, su uso está en auge debido a su rol fundamental en plataformas digitales como Uber, Airbnb o Deliveroo. No obstante, dicho sistema de evaluación no es exclusivo de las plataformas digitales y no es difícil imaginar su implementación en empresas tradicionales, por lo que estos sistemas podrían convertirse en un fenómeno de rápida expansión en los próximos años1.

En resumen, este tipo de sistema de evaluación se basa en que el cliente evalúa su satisfacción con el servicio prestado por un trabajador mediante una escala de puntuación, normalmente de una a cinco estrellas. Esta puntuación servirá para evaluar al trabajador y de ella dependen aspectos tan importantes como la retribución o incluso la continuidad del trabajador en la empresa. El ejemplo más visible de las consecuencias que este sistema puede tener para el trabajador es el conductor de Uber que es expulsado de la aplicación porque su valoración (rating) ha descendido el umbral mínimo del 4,6 sobre una puntuación total de 52 o, en el sentido contrario, el trabajador de Uber al que se le ofrece una retribución especial por su buena puntuación.3

Este sistema ofrece a las Empresas dos ventajas especialmente significativas:

1 Ducato, Kullman Y RocA, "Customer Ratings as a Vector for Discrimination in Employment Relations? Pathways and Pitfalls for Legal Remedies". Proceedings of the Marco Biagi Conference 2018 (January 31) p. 2

2 UBER, "Preguntas $\quad$ Frecuentes" (https://www.uber.com/esCL/drive/arica/resources/preguntasfrecuentes/)

3 ROSENBLAT, LEVY, BAROCAS AND HWANG, "Discriminating Tastes: Uber's Customer Ratings as Vehicles for Workplace Discrimination”, 9 Policy and Internet 3, 2016 p. 256-279 p.6. 
En primer lugar, permite que los clientes vean las opiniones de los otros clientes sobre los servicios que presta un trabajador, lo que incentiva la creación de un entorno de confianza entre el cliente y la empresa que es especialmente necesario para plataformas digitales de nueva creación que, en sus inicios, no gozan de una amplia reputación en el mercado.4

En segundo lugar, este sistema posibilita la gestión automática o semi-automática de la plantilla, lo que permite a la empresa prescindir de muchos puestos de trabajo que serían necesarios para evaluar, recompensar y promocionar a los trabajadores5. Esta ventaja es especialmente interesante para empresas que cuentan con una plantilla de colaboradores/trabajadores muy dispersa, como es el caso de ciertas plataformas digitales, como por ejemplo Uber.6

Sin este sistema, sería mucho más difícil que Uber gestionara su plantilla de más de 3 millones de conductores7, o que GLOVO evaluara y compensara a sus ciclistas o que un cliente de Upwork le encargara una tarea a uno de sus profesionales sin conocer nada acerca de esa persona.

No obstante, existen varios estudios que apuntan que las evaluaciones de los clientes podrían contener sesgos discriminatorios. El objeto de este artículo es analizar si se produciría una vulneración del principio de igualdad y del principio de no discriminación en el supuesto de que estas evaluaciones se tuvieran en cuenta para la toma de decisiones de carácter laboral. 8

Si esto sucediera, el uso de este tipo de sistemas de evaluación supondría un riesgo para la protección de estos colectivos ya que el sistema de evaluación aparentemente objetivo, que solamente tiene en cuenta la opinión de los clientes, podría en realidad blanquear decisiones legalmente discriminatorias9. Asimismo, este fenómeno podría extenderse al resto de empresas de servicios que no son plataformas digitales gracias al aumento de la utilización de la tecnología en la sociedad. En este caso, este fenómeno

\footnotetext{
4 DUCATO, Kullman Y Roca, op. cit., p. 8.

5 DUCATO, KullMan Y RocA, OP. CIT., P. 2.

6 ROSENBLAT, LEVy, BAROCAS AND HWANG, op. cit., p.3.

7 MANSOOR IQBAL, "Uber Revenue and Usage Statistics (2018)" Businessofapps, 2018 (http://www.businessofapps.com/data/uber-statistics/).

8 Rosenblat, LeVy, Barocas And HwANG, op. cit. ; DuCATO, Kullman Y RocA, op. cit.; BartletT y Gulati. (2013) “Discrimination by Costumers” Iowa Law Review, Vol. 102:223 p. 224-257.

9 BArocas Y SelbSt, "Big Data's Disparate Impact", 104 Calif. L. Rev. 671, 2016, p. 1382; PASQUALE,

“The Black Box Society", Harvard University Press, 2015, p. 41.
} 
afectaría a una cantidad muy superior de trabajadores, por lo que su estudio es claramente relevante. 10

Vaya por delante que algunos de los ejemplos que se citan como Uber o Airbnb, se aportan únicamente con el objetivo de comprender el funcionamiento de los sistemas de evaluación para analizar qué problemáticas legales podrían surgir de su uso en la evaluación de trabajadores. Es importante tener en cuenta que este artículo no pretende analizar la consideración de trabajadores o no de los colaboradores de dichas plataformas y tampoco pretende concluir si esos sistemas funcionan de una determinada manera en la actualidad en España, ya que normalmente la información que tenemos de esas plataformas proviene de su funcionamiento en Estados Unidos y, por razones obvias, desconocemos del funcionamiento interno de estos sistemas en España.

Nuestro objetivo actual es reflexionar acerca de las problemáticas que surgirían del uso de sistemas de este tipo en la evaluación de trabajadores y no analizar si la conducta específica de una Empresa contraviene o no lo establecido en el ordenamiento jurídico español. La estructura de este artículo consiste en una breve explicación sobre estos sistemas de evaluación y los posibles sesgos que se producen en las evaluaciones, un análisis sobre la jurisprudencia en materia de discriminación en España y una conclusión acerca de la existencia o no de riesgos legales en estos sistemas de evaluación junto con una propuesta de mejora de este tipo de sistemas de evaluación.

Para ello analizaremos la bibliografía actual sobre estos sistemas de evaluación y su posible discriminación, así como la jurisprudencia más reciente sobre este aspecto, especialmente la jurisprudencia europea sobre discriminación.

\section{Los sistemas de evaluación}

\section{1. ¿Por qué existen y en qué consisten estos sistemas de evaluación?}

"Solo existe un jefe: el cliente". Ésta célebre cita pertenece a Sam Walton, creador de Wal-Mart, la cadena de supermercados más grande del mundo11 y responde a una filosofía de negocio que se enmarca dentro de una corriente de marketing denominada "Customer Oriented Strategy", que entiende que las empresas no deben orientarse ni a los accionistas, ni a los productos, sino que deben enfocar todas sus decisiones al 10 LIU, "A new job search app allows restaurant employees and employers to rate each other", The Star, 2017, (https://www.thestar.com/life/food_wine/2017/10/28/a-new-job-search-app-allowsrestaurantemployees-and-employers-to-rate-each-other.html).

11 Deloitte Studie, "Global Powers of Retailing" [archivo PDF], 2018, p. 10 (https://www2.deloitte.com/content/dam/Deloitte/at/Documents/about-deloitte/global-powers-ofretailing-2018.pdf). 
cliente, que es el que a través de su decisión de compra, acabará teniendo un impacto sobre el éxito o el fracaso económico de la empresa.12 En España, Mercadona define su filosofía de empresa de forma similar. 13

Atendiendo a esta filosofía, el desempeño del trabajador debería adaptarse a las exigencias del cliente y, por lo tanto, su evaluación debería basarse en el cumplimiento o no de las necesidades del cliente. Tradicionalmente, han existido mecanismos para conocer la opinión de los clientes sobre los productos comprados o los servicios prestados. Ejemplos de ello son las encuestas de calidad en las habitaciones de hoteles, las encuestas posteriores a una llamada en los call-centers de atención al cliente o, de forma más rudimentaria, las propinas que dejan los clientes en un restaurante. No obstante, la aparición de nuevas tecnologías y el incremento de su uso cotidiano permite que las empresas obtengan de forma sencilla y fiable una cantidad muy superior de información sobre la satisfacción de un cliente con la prestación de servicios realizada por un trabajador en concreto. 14

Un ejemplo de esto se puede observar en los negocios tradicionales de atención al público. Anteriormente, las empresas podían realizar encuestas de satisfacción entre sus clientes, pero ese procedimiento era lento, costoso y poco fiable. Actualmente empresas como El Corte Inglés o Cinesa han implantado un pequeño poste conectado a internet en el que el cliente solamente tiene que escoger entre cinco caras, de verde y feliz, a roja y enfadada, con las que indican la satisfacción del cliente con la atención del trabajador. 15

El ejemplo más visible del auge de la utilización de la tecnología en este sentido es el sistema de evaluación de 5 estrellas implantado en muchas empresas y, en especial, en empresas basadas en plataformas digitales. Este sistema permite que un cliente de Uber evalúe su conductor al terminar su viaje, o que un cliente de Airbnb valore la hospitalidad de su anfitrión una vez terminada la estancia. De estas valoraciones dependen ciertos incentivos económicos del colaborador, la cantidad de tareas que se le van a asignar o incluso su continuidad en la aplicación.16

12 DAlgic, DeniCERI, "Customer Oriented Marketing Strategy", American Marketing Association https://www.ama.org/Documents/customer-oriented-marketing-strategy-theory-and-practice.pdf

13 El principal objetivo de Mercadona es que el Jefe (cliente) esté siempre satisfecho MERCADONA, "Modelo", 2018 (https://info.mercadona.es/es/conocenos/modelo/el-jefe).

14 ZUIL M., “¿Cuántas estrellas merece mi mileurista? La moda de puntuar trabajadores llega a España.”, El Confidencial, https://www.elconfidencial.com/empresas/2018-02-04/puntuar-valorar-trabajadores-elcorte-ingles_1515921/.

15 Ibídem

16 Rosenblat, LeVy, Barocas And HWANG, op. cit., p. 5. 
De esta manera, la compensación de los trabajadores está perfectamente ajustada a la percepción de calidad del servicio del cliente. Los trabajadores tienen un incentivo económico directo en que su prestación de servicios logre la máxima puntuación posible por parte del cliente. En sentido contrario, los trabajadores tienen un desincentivo económico directo en que su prestación no sea valorada con la máxima puntuación.17

En cierto modo, se consigue que el trabajador asuma casi por completo la responsabilidad de la prestación de servicios, ya que la valoración del cliente repercutirá en su remuneración y en su continuidad en la aplicación. Se pretende que ese aumento de la responsabilidad del trabajador se convierta en un aumento de la calidad de su prestación de servicios. 18

La Sentencia 53/2019 del Juzgado de lo Social, en el marco de un despido en la empresa GLOVO, ofrece un buen resumen de lo previamente descrito: "las TIC permiten acceder a un gran volumen de información y a su tratamiento rápido y barato mediante la creación de los correspondientes algoritmos que a través de la elaboración de perfiles acceden al conocimiento detallado de cuándo, cómo, dónde y con qué resultado se ha trabajado. Todo ello a su vez permite vincular la retribución compensatoria del trabajo, no al tiempo en que el trabajador está a disposición sino al resultado de su actividad. El salario fijo da paso al salario variable en función del resultado de la actividad personal del trabajador, lo que también supone en buena medida la traslación a éste de lo que hasta ahora venían siendo riesgos que asumía el empresario al adjudicarse el trabajo que le era prestado."

Para el objetivo del presente trabajo, tomaremos como modelo de referencia el sistema de evaluación utilizado por Uber en Estados Unidos porque es el que ha sido estudiado con más detalle. A los efectos de nuestro análisis, asumiremos que su funcionamiento es similar al de otras aplicaciones y analizaremos el impacto que tendría este sistema en la evaluación de trabajadores, sin entrar a discutir la concreta calificación jurídica de los conductores de Uber.

El sistema consiste en la calificación por parte del cliente de una prestación de servicios. Después de cada viaje, el pasajero de Uber valora con una puntuación de una a cinco estrellas la calidad del servicio prestado y eso genera automáticamente una puntuación general del conductor. El pasajero también tiene la posibilidad de dejar algún comentario acerca de su valoración. Sin embargo, esto no es obligatorio de forma que el cliente no tiene por qué justificar su valoración 19

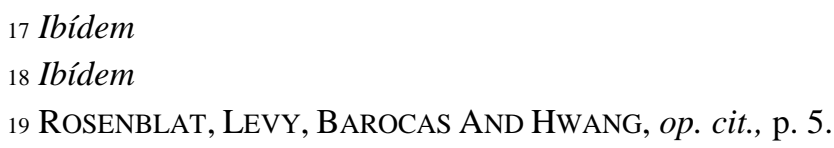


Hay otras aplicaciones, como por ejemplo Airbnb, que solicitan una justificación más específica de la valoración, solicitando una puntuación por apartados (limpieza, hospitalidad etc...) y una justificación descriptiva ante una valoración negativa. No obstante, igual que en el caso de Uber, el cliente no tiene la obligación de ofrecer una justificación si desea no hacerlo.20

En el sistema de Uber, las valoraciones son confidenciales, de forma que el conductor no puede saber qué cliente le valoró positivamente y qué cliente le valoró negativamente. Cuando la puntuación de un conductor se sitúa por debajo de la media de los conductores, éste recibe mensajes de Uber con los comentarios que haya efectuado alguno de sus pasajeros y recomendaciones de carácter general dirigidas a los transportistas de la zona, para que puedan mejorar su prestación de servicios.21

En este momento, debemos analizar las consecuencias iniciales que la configuración de dicho sistema tendría para los trabajadores que fueran evaluados por un sistema como el descrito. En primer lugar, si los clientes no indican las razones de su baja valoración, se puede dar la situación de que un trabajador no conozca quién le ha valorado negativamente ni por qué. Esto puede dejar al conductor en una posición muy delicada por dos motivos. En primer lugar, porque al desconocer qué cliente le ha evaluado negativamente y el motivo de su evaluación, no tiene forma de entender qué acción ha hecho incorrectamente y, por lo tanto, no tendrá forma de mejorar, solamente podrá intentar adivinar qué fue lo que incomodó a su cliente.

Este es el caso de Uber dónde, según un estudio reciente, la mayoría de los conductores sienten ansiedad y frustración ante las evaluaciones de los pasajeros por no saber qué había cambiado en su forma de conducir entre una evaluación positiva y una negativa.22

Al no saber sobre qué se les está evaluando, muchos conductores de Uber prefieren compensar la posible e impredecible mala evaluación por parte de un cliente ofreciendo agua, comida, cargador de batería y teniendo una actitud mucho más atenta y servicial que la que normalmente tendría otro trabajador o un taxista. 23

La segunda forma mediante la cual el sistema de evaluación puede provocar indefensión al trabajador es la imposibilidad del trabajador de rebatir la opinión del cliente. El sistema asume que el cliente siempre tiene la razón y, aunque desde un punto de vista

20 AIRBNB, “Ayuda de viaje: Evaluaciones", 2018 https://www.airbnb.es/help/article/1257/how-do-starratings-work.

21 Rosenblat, Levy, Barocas And Hwang, op. cit., p. 4.

22 Ibídem

23 Ibídem 
comercial eso puede tener sentido, esta premisa puede dejar al trabajador en una situación de absoluta indefensión. Imaginemos, por ejemplo, que el pasajero le ha pedido al conductor que condujera a una velocidad temeraria porque llegaba tarde a su destino. Si el conductor no sigue las instrucciones del pasajero, lo más probable es que reciba una valoración negativa sin que el conductor pueda rebatir dicha valoración justificando su conducta.

No obstante, este artículo se pretende centrar en el estudio de estos sistemas desde la perspectiva del principio de igualdad y el principio de no discriminación, por lo que una vez apuntada esta interesante cuestión, no entraremos a analizar si de ella derivaría la indefensión del trabajador y si, en su caso, esto supondría la ilicitud de estos sistemas.

La valoración efectuada por parte de los clientes afecta en cuanto a la continuidad del conductor en la aplicación y en cuanto a la remuneración de éste. Si la nota media del conductor se sitúa por debajo de una media mínima de aproximadamente 4,6 el conductor recibirá un mensaje alertando de que corre el riesgo de ser desactivado de la aplicación temporalmente. Si su puntuación no mejora durante los próximos días, la aplicación le suspenderá temporalmente. Esta media límite varía por zonas y por tipo de servicio. 24

En cuanto a la remuneración, los conductores con mayor puntuación tienen preferencia en la recepción de viajes a realizar, por lo que un conductor con una puntuación mayor pasará por delante de un conductor con una puntuación inferior frente a una solicitud de un cliente25. Si la demanda es baja, eso repercute en la remuneración del trabajador. Asimismo, Uber a veces ofrece condiciones especiales a los conductores con las puntuaciones más altas, de forma que se les garantiza un salario mínimo por hora a esos trabajadores si cumplen unos criterios mínimos de aceptación de pedidos, de horas trabajadas y de valoración por parte de los clientes. De modo que la puntuación influye en si se le ofrece o no esa garantía a un conductor.26

Una vez resumido el funcionamiento del sistema de evaluación, vemos que éste no responde a unas características específicas de Uber o de Airbnb, sino que este mismo modelo podría adaptarse a cualquier tipo de empresa, especialmente aquellas que traten de forma directa con el público. En este sentido, en el siguiente apartado analizaremos qué tipo de empresas usan o podrían usar este tipo de sistemas de evaluación

2.2. ¿Qué empresas usan este sistema?

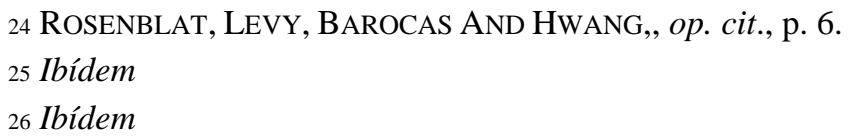


Como ya hemos apuntado anteriormente, las empresas que están usando de forma mayoritaria este tipo de sistemas de evaluación son las plataformas digitales que normalmente empezaron siendo proyectos "Start-Up" irrumpiendo en un mercado tradicional, como Uber, Airbnb o Deliveroo.

No obstante, también son aplicables estos sistemas a las empresas tradicionales como $E l$ Corte Inglés, Cinesa o empresas de teleoperadores. En este sentido, hay muchos servicios telefónicos de atención al cliente que usan sistemas evaluativos similares a los de Uber, basándose en la puntuación ofrecida por los clientes justo después de la llamada.

A esos sistemas evaluativos también les son aplicables las características descritas en al apartado anterior y, además, tienen una problemática añadida para sus trabajadores y es que muchas veces el cliente les va a evaluar a ellos valorando su satisfacción global con los servicios de la empresa y no su satisfacción con el servicio prestado por el trabajador que les atiende la llamada27. En este caso, si las evaluaciones fueran la causa de un despido disciplinario, se estaría imputando al trabajador unos resultados que en realidad corresponderían a la empresa, por lo que se estaría sancionando al trabajador por una conducta ajena a su responsabilidad.

También es relevante tener en cuenta que las empresas de plataformas digitales están en una fase inicial de crecimiento basada en la prueba y error. Por lo tanto, su afectación hoy en día puede ser menor, pero su crecimiento en los próximos años puede ser muy relevante. A modo de ejemplo, Uber al principio se dedicaba solamente al transporte de personas. 28 Sin embargo, actualmente también se dedica al reparto de comida y en poco tiempo Uber planea ofrecer un servicio similar al de las ETT para camareros y guardias de seguridad hecho que a su vez comportará que otras empresas se planteen competir con Uber imitando los aspectos que le permiten tener una ventaja competitiva. 29

Finalmente, la utilización de estos sistemas de evaluación ha excedido el ámbito de la empresa y ya existen distintas aplicaciones que permiten a los clientes valorar a trabajadores de distintas empresas por su prestación de servicios. 30

\footnotetext{
27 ZUIL M., op. cit.

28 Del Castillo C., "Uber planea 'uberizar' a camareros, azafatas o guardas de seguridad con un servicio similar al de las ETT”, El Diario, 2018 (https://www.eldiario.es/tecnologia/Uber-servicio-ETTscamareros-seguridad_0_827667615.html)

29 Ídem

30 Todolí A., op. cit.
} 
Un ejemplo de esto es "Guudjob", una start up creada en Madrid que permite a los clientes valorar tanto autónomos como trabajadores. La descripción del funcionamiento
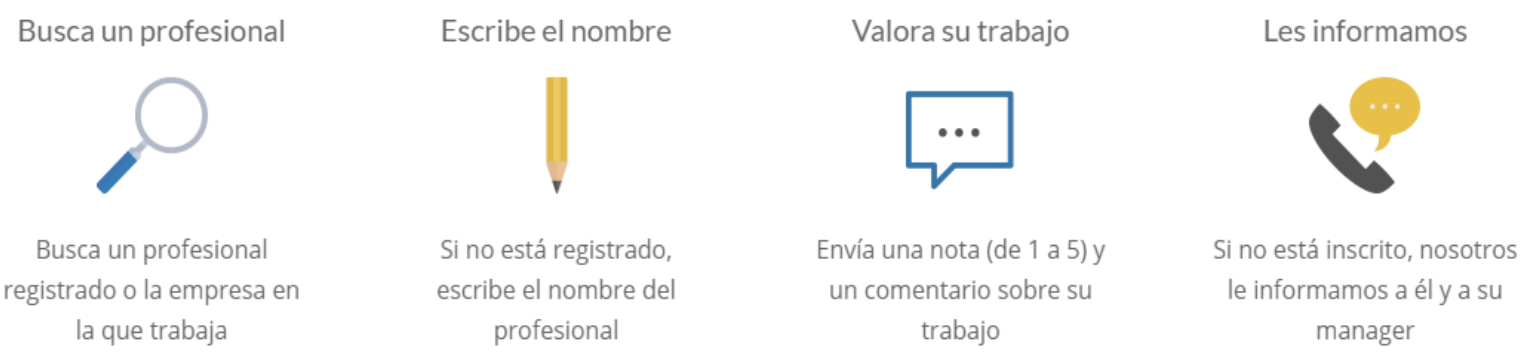

de esta aplicación es un ejemplo magnífico de cómo operan estos sistemas de evaluación y está ilustrado en la web de la aplicación de la siguiente manera31:

Como se puede observar, esta aplicación va más allá de los sistemas previamente descritos, ya que permite la evaluación de trabajadores en empresas que no tengan el sistema de evaluación implantado e incluso cuando el trabajador no se haya inscrito en la aplicación. Esto podría entrañar otras problemáticas jurídicas, especialmente en materia de Protección de Datos, que escapan del análisis de este trabajo. No obstante, es relevante citar este ejemplo para observar la complejidad que estos fenómenos conllevan.

Las implicaciones que este funcionamiento puede tener en cuanto a la escalabilidad de este modelo son muy importantes, ya que la empresa no necesitaría crear y controlar un sistema de evaluación de los trabajadores interno, si no que podría acudir a las evaluaciones contenidas en esta aplicación y usarlas en la evaluación de sus trabajadores.

Antes de pasar al siguiente apartado sobre la existencia o no de discriminación, vale la pena remarcar que estos sistemas no son solamente un fenómeno dirigido a la evaluación de trabajadores. "Guudjob" se promociona como una herramienta innovadora para revolucionar la forma de hacer marketing (con el conocimiento exacto de las necesidades de los clientes), operaciones (por la rapidez con la que estos datos puede obtenerse y cambiar la producción o la prestación de servicios de la empresa) y la gestión de datos (por la gran cantidad de datos y la calidad de estos para el negocio).32

\section{3. ¿Existen sesgos en las evaluaciones de los clientes?}

${ }_{31}$ GuUdJOB, "Página web de Guudjob” (https://www.guudjob.com)

32 https://www.guudjob.com 
Como hemos visto, estos sistemas de evaluación dependen prácticamente en su totalidad de la opinión del cliente. Esta opinión nunca estará libre de sesgos, ya que las personas estamos continuamente influenciadas por sesgos cognitivos que alteran nuestra manera de comprender la realidad y nos influencian en nuestra toma de decisiones. Por ejemplo, nuestro cerebro tiende a valorar a una persona por la primera información que tenemos de ella, siendo muy complicado cambiar la opinión que la primera impresión de esta persona nos ha formado.33

Es especialmente relevante para este trabajo conocer si, dentro de estos sesgos que inevitablemente tenemos todas las personas, existe un sesgo de carácter discriminatorio en las evaluaciones de los clientes por el cual éstos evalúan peor a los trabajadores que pertenecen a un determinado colectivo históricamente discriminado.

La forma más idónea para analizar esta cuestión sería disponer de datos sobre las evaluaciones de los clientes y las características de los trabajadores evaluados. No obstante, aún no existe ningún estudio que haya tenido acceso a estos datos, por lo que solamente podremos acceder a estudios realizados en situaciones similares donde el consumidor evalúa su grado de satisfacción con los servicios prestados: en plataformas como Airbnb, estudios sobre los hábitos de compra de los consumidores en internet o evaluaciones que hagan los clientes de una empresa sobre sus comerciales.

A partir de estos estudios podremos sacar conclusiones sobre si las evaluaciones de los clientes contienen sesgos discriminatorios o no. Por ejemplo, uno de estos estudios sobre la venta de Ipods en internet demostró que cuando en la foto aparecía una mano de piel negra había menos solicitudes de compra y a un precio inferior que cuando aparecía una mano blanca.34 Otro estudio muy similar se basaba en el estudio de las ventas de cromos de baseball, cuando la mano que aparecía en la imagen era de piel negra y no blanca, el cromo se vendía por un precio un $20 \%$ inferior. 35

En cuanto a la plataforma Airbnb, un estudio demostró que los clientes estaban dispuestos a pagar un $12 \%$ más a los huéspedes que no eran afroamericanos por el alquiler de propiedades equivalentes y evaluaban peor a los huéspedes afroamericanos por la ubicación de sus propiedades. 36

33 KAHnEMAN D. , “Thinking, fast and slow”. New York: Farrar, Straus and Giroux. 2011.

34 DolEAC AND STEIN, "The visible hand: Race and online market outcomes." The Economic Journal 123, no. 572 (2013): 469-492.

35 AYRES, BANAJI AND JOLLS, "Race effects on eBay." The RAND Journal of Economics 46, no. 4, 2015 : 891- 917.

36 Edelman AND LucA, "Digital Discrimination: The Case of Airbnb.com", 9 Harvard Bus. Sch. Negotiation, Orgs. \& Mkts. Unit, Working Paper No. 14-054, 2014. 
Otro estudio realizado sobre 1000 taxis en New Heaven, (Connecticut) observó que los taxistas afroamericanos recibían aproximadamente un tercio menos en propinas por cada viaje que sus compañeros no afroamericanos.37 Asimismo, se comprobó una situación similar en las propinas a camareras respecto de los camareros.38

No obstante, para el propósito de este trabajo, el estudio más interesante es el que se hizo sobre las evaluaciones por los clientes de los comerciales de la empresa de marketing WordStream. Cerca de 300 clientes de esta empresa evaluaron el grado de satisfacción con el trabajo efectuado por el representante de WordStream con el que trabajaban. A pesar de que en términos generales las representantes mujeres tenían más experiencia y sus cuentas de clientes tenían un rendimiento superior al de sus compañeros hombres, el resultado fue que los clientes evaluaron un $21 \%$ peor a las representantes mujeres que a los representantes hombres.39

Este estudio además analizó si este sesgo afectaba solamente a los clientes del sexo masculino. Sin embargo, este estudio concluyó que en las evaluaciones por parte de las clientes del sexo femenino existía una brecha más amplia entre la evaluación de la representante y el rendimiento y experiencia real de ésta, por lo tanto, el sesgo discriminatorio existía en ambos sexos.40

En el mismo sentido, se han realizado muchos estudios sobre las valoraciones de los profesores de universidad por parte de los alumnos que evidencian que los alumnos valoran peor a miembros de colectivos históricamente discriminados en dichas evaluaciones. 41

Todos estos estudios parecen indicar que efectivamente los consumidores discriminan tanto en sus decisiones de compra, como en sus valoraciones sobre los servicios prestados. No obstante, para evaluar los posibles riesgos que de este sistema se deriven,

37 AYres ET AL. “To Insure Prejudice: Racial Disparities in Taxicab Tipping”, 114 Yale L.J. 1613, 1616 (2005)

38 PARRET, "Customer Discrimination in Restaurants: Dining Frequency Matters", 32, J. Lab. Res. 87, 100-03 (2011).

39 Covert B., "Female Client Service Reps Get Lower Scores Despite Better Performance and Experience", THINKPROGRESS (May 22, 2014), https://thinkprogress.org/female-client-service-reps-getlower-scores-despite-better-performance-and-experience-dfa457062f5c/.

40 KIM L., "Gender Bias in Online Marketing: Data Shows Women Are Undervalued by 21\%", Wordstream Blogg, 2018 https://www.wordstream.com/blog/ws/2014/05/13/gender-bias.

41 GutiÉrRez Et Al., "The intersections of Race and Class and Woman in Academia" Utah State University Press, 2012. 
debemos analizar primero el concepto de discriminación y su interpretación por parte de los tribunales españoles y europeos.

\section{3. ¿Cómo afectan estos sistemas a los principios de igualdad y no discriminación?}

\section{1. ¿Existe una vulneración del derecho a la igualdad y a la no discriminación?}

En primer lugar, analizaremos si los sistemas de evaluación estudiados en el presente trabajo vulneran el derecho a la igualdad establecido en el artículo 14 CE. En este sentido, debemos recordar que la empresa vulnera este derecho cuando ante una situación de igualdad, trata de forma desigual a un trabajador sin que exista una justificación razonable (STC 22/1981, de 2 de julio núm. rec. 223/1980).

En el presente supuesto, la empresa está aplicando a todos los trabajadores el mismo sistema evaluativo, de forma que no se está tratando a los trabajadores de forma desigual. En cuanto a las posibles desigualdades retributivas que puedan surgir de la aplicación de dicho sistema de evaluación, la empresa puede razonablemente argumentar que estas diferencias están justificadas en las preferencias de los clientes.

Como es conocido, la retribución desigual entre dos trabajadores no vulnera por sí sola el derecho a la igualdad (STC 34/1984 de 9 de marzo núm. rec. 539/1983). Es por eso que, en el improbable caso de que en una empresa no existieran trabajadores susceptibles de ser discriminados por alguno de los motivos legalmente previstas, la aplicación de este tipo de sistemas no supondría, en principio, una vulneración del derecho a la igualdad.

En cuanto a la vulneración del principio de no discriminación, debemos analizar si los sesgos en las valoraciones de los analizadas en este artículo responden a las causas discriminatorias previstas. En este sentido, hemos tratado estudios que analizan sesgos por razón de género42 y por razón de raza43, ambas causas de diferenciación prohibidas por el principio de no discriminación. En consecuencia, procede analizar si existe discriminación tanto directa como indirecta.

Resulta difícil argumentar que las empresas que aplican estos sistemas estén discriminando de forma directa a sus trabajadores, ya que estos sistemas no diferencian a los trabajadores por sexo o por raza. Del mismo modo, las empresas aplican de forma uniforme los resultados obtenidos por el algoritmo sin hacer diferenciación alguna por

42 COVERT, op. cit.; PARRET, op. cit.; GUTIÉRREZ et al, op. cit.

43 Doleac and STEIn, op. cit.; AYRES, BANAJI \& JOlls, op. cit.; EdElMAN AND LuCA, op. cit.; AYRES ET AL, op. cit. 
las causas de discriminación mencionadas. En este sentido, el sistema evaluativo es aparentemente neutro.

No obstante, sí que existe discriminación directa en las valoraciones de los clientes. Como ha quedado probado en los estudios aportados, los clientes valoran de forma menos favorable a los trabajadores por razón de sexo y por razón de raza. Sin embargo, esta conducta no es jurídicamente ilícita en el sentido de que los clientes no tienen una obligación legal de no discriminar en sus valoraciones. Además, una posible regulación en este sentido sería inmensamente controvertida. 44

Aun así, ello significa que una parte de los datos sobre los que funciona el algoritmo de este sistema de evaluación provienen de una discriminación directa por parte de los clientes. Esto nos lleva a analizar si la empresa está incurriendo en una discriminación indirecta por usar esos datos que provienen de una discriminación directa.

Para hacerlo, debemos partir de la base de que estos sistemas son creados por personas y funcionan mediante datos expresados por personas. Así pues, aunque un algoritmo pudiera parecer un método exento de cualquier tipo de subjetividad, no debemos olvidar que las personas que crean los algoritmos en los que se basan estos sistemas tienen sesgos y, aún más importante, que la información con la que operan también puede contener sesgos, de modo que los algoritmos también son susceptibles de ser discriminatorios. 45

En el presente caso, eso es aún más visible, ya que la información con la que funciona el algoritmo proviene de una discriminación directa $\mathrm{y}$, por lo tanto, el resultado que surja de las operaciones que realice el algoritmo continuará siendo discriminatorio.46 No nos parecería lógico sostener que, por el hecho de introducir los datos en un algoritmo aparentemente neutro, una información que proviene de las valoraciones de unos clientes que discriminan de forma directa se convierta en una información libre de discriminación.

Por lo tanto, un sistema de evaluación como el descrito en el presente trabajo presentaría resultados discriminatorios, mostrando valoraciones desproporcionadamente adversas para los trabajadores de grupos históricamente discriminados 47 .

44 BARTLETT AND GulatTi, op. cit. p. 238.

45 Mittelstad Et Al., "The ethics of algorithms: Mapping the debate", 3 Big Data \& Society 1, 2016, p.1.

46 Ducato, Kullman and Roca, op. cit.

47 BAROCAS AND SELBST, op. cit. p. 673. 
No obstante, cabe valorar si eso implicaría per se la existencia de discriminación por parte del empresario. Para hacerlo debemos analizar si aplicando este sistema de evaluación, ante una prestación de servicios de igual valor, la empresa perjudicaría a un trabajador por alguna de las causas mencionadas.

Así pues, si los clientes evaluaran de la forma que se ha descrito en el presente trabajo, éstos evaluarían peor a una mujer o a un inmigrante ante una prestación de igual valor. Esta peor evaluación resultaría en una puntuación inferior en el sistema evaluativo y ello, como hemos expuesto anteriormente, repercutiría en una menor retribución, en unas posibilidades inferiores de acceder a determinados beneficios o incluso en un mayor riesgo de ser desconectados de la aplicación.

En conclusión, parece razonable afirmar que, si el empresario se basara en el sistema evaluativo para tomar ese tipo de decisiones laborales, la empresa estaría indirectamente discriminando al trabajador, siendo la consecuencia final un trato distinto dependiendo de si el trabajador se encuadra en alguna de las mencionadas causas de discriminación.

Eso sería así, incluso en el caso de que la empresa no fuera la creadora del sistema de evaluación, sino que se basara en un sistema de evaluación externo, como por ejemplo sería el caso de Guudjob. En este sentido, cabe recordar que la existencia o no de discriminación no está vinculada a un ánimo discriminatorio por parte de la empresa si no que se analiza la neutralidad de la medida tomada. 48

Si se apreciara la existencia de discriminación indirecta, sería interesante reflexionar si el empresario estaría obligado a realizar un proceso previo de comprobación sobre la forma en la que se toman esos resultados y de análisis de los mismos. Con este proceso de due dilligence, la empresa podría saber si la aplicación de ese sistema en su organización está creando resultados discriminatorios o no.

Una vez concluido que, en principio, la aplicación de un sistema de estas características supondría una discriminación indirecta, es procedente acudir a la jurisprudencia para ver si se ha enjuiciado algún supuesto similar y, si en esos casos, existe alguna razón objetiva que justifique la discriminación.

Sin embargo, debido al carácter novedoso de estos sistemas, no existe aún ninguna Sentencia que haya resuelto un supuesto idéntico al analizado a la fecha de elaboración de este trabajo. No obstante, existen algunos casos que pueden resultar interesantes para el estudio de esta cuestión y que pueden ofrecernos indicios de cómo se podría enfocar una cuestión de esta naturaleza en el futuro.

${ }_{48}$ GARCías SAlas A.I, op. cit., p. 261. 
Para no extendernos de forma innecesaria, procederemos a resumir las cuatro sentencias que consideramos relevantes para el caso concreto y dejamos a elección del lector la posibilidad de consultarlas para un análisis más profundo de la cuestión.

El primer caso que analizaremos es la STJUE de 10 de julio de 2008 (TJCE 2008\157) Caso Feryn que versa sobre una empresa belga de instalación de puertas que en una entrevista afirmó que no querían contratar a trabajadores marroquíes ya que sus clientes no los querían y ellos tenían que trabajar "como el cliente quiera". La STJUE declaró que la actuación de la empresa había sido discriminatoria. El Tribunal no se pronunció expresamente sobre si el hecho de que la empresa afirmara que se había tomado esa medida debido a las exigencias de los clientes era una justificación válida para la misma. No obstante, del fallo de la Sentencia se deduce que el Tribunal no lo consideró así.

Un caso parecido a éste es el que enjuició en fecha 22 de diciembre de 1987 (RTC 1987 207) el Tribunal Constitucional. En ese caso la controversia surgía de la opción que ofrecía IBERIA a las mujeres de 35 y 40 años que eran auxiliares de vuelo de retirarse anticipadamente y que no ofrecía a los auxiliares de vuelo hombres debido a "las especiales condiciones físicas que en todas las compañías aéreas exigen los usuarios a las mujeres que prestan sus servicios como Auxiliares de Vuelo." Aunque el TC no entra expresamente a decidir si las exigencias de los clientes son causa justificativa o no, la STC admite que hay trabajos en los que la presencia física puede ser determinante, pero no en este caso, y menos cuando solo se exige a las mujeres y no a los hombres.

Otra Sentencia que es interesante para nuestro análisis es la STJUE de 14 de marzo de 2017 (TJCE 2017 24) Caso Bougnaoui. Este caso trata de una trabajadora que prestaba servicios en las oficinas de los clientes y que pasó a llevar velo islámico. No obstante, un cliente se quejó exigiendo que "no hubiera velo la próxima vez”. A raíz de estas quejas, la Empresa exigió a la trabajadora que se quitara el velo durante las horas de trabajo, a lo que la trabajadora se negó, razón por la cual fue despedida. La conclusión del TJUE fue la siguiente: "La voluntad de un empresario de tener en cuenta los deseos de un cliente de que los servicios de dicho empresario no sigan siendo prestados por una trabajadora que lleva un pañuelo islámico no puede considerarse un requisito profesional esencial y determinante en el sentido de esta disposición."

El último caso que analizaremos es la STJUE de 14 de marzo de 2017 (TJCE 2017\23) Caso Achbita. Este caso es muy similar al anterior en cuanto a que se trata de la prohibición a una trabajadora trabajar con el pañuelo islámico cuando en la Empresa existía una política de neutralidad en los símbolos que podían llevar los trabajadores. El 
Tribunal resuelve que no existe una discriminación directa, pero obiter dicta no descarta que pueda existir una discriminación indirecta en el caso que dicha política de neutralidad en los símbolos perjudicara a una mayoría de trabajadores por una de las causas de discriminación. Sin embargo, el TJUE dice que no existirá discriminación " $s i$ puede justificarse objetivamente con una finalidad legítima y si los medios para la consecución de esta finalidad son adecuados y necesarios".

En este sentido el Tribunal dice que "en cuanto al requisito de la existencia de una finalidad legítima, procede señalar que ha de considerarse legítima la voluntad de seguir un régimen de neutralidad política, filosófica o religiosa en las relaciones con los clientes tanto del sector público como del sector privado." Aún resulta más interesante para nuestro estudio lo que argumenta a continuación el Tribunal: "En efecto, el deseo de un empresario de ofrecer una imagen neutra ante sus clientes está vinculado a la libertad de empresa, reconocida en el artículo 16 de la Carta, y tiene, en principio, un carácter legítimo, en particular cuando el empresario sólo incluye en la persecución de esa finalidad a los trabajadores que, en principio, van a estar en contacto con sus clientes."

Por lo que, por el momento, parece que el TJUE ha dejado claro que la voluntad de cumplir con los deseos del cliente no es un requisito profesional esencial y determinante. No obstante, en Achbita, STJUE de 14 de marzo de 2017 (TJCE 2017 23), se puede apreciar un pequeño matiz o cambio jurisprudencial, cuando se reconoce que la voluntad del empleador de mantener una apariencia de neutralidad ante el cliente forma parte del derecho fundamental a la libertad de empresa. En ese caso, se podría defender analógicamente, que la voluntad del empleador de mantener una apariencia de adaptabilidad a las preferencias del cliente también forma parte del derecho fundamental a la libertad de empresa. Aunque con la doctrina de los casos Feryn, STJUE de 10 de julio de 2008 (TJCE 2008\157), y Bougnaoui, STJUE de 14 de marzo de 2017 (TJCE 2017\24), no parece una justificación muy sólida.

Siguiendo con esta argumentación, en el caso de la aplicación de sistemas de evaluación basados en las evaluaciones de los clientes, cabe valorar si existirían otras justificaciones vinculadas al principio de libertad de empresa. En este sentido, parte de la doctrina ha discutido acerca del valor justificativo que puede tener el hecho de que la empresa busque un nivel de rentabilidad o de viabilidad económica. Esta doctrina ha afirmado que, si bien es cierto que una mayor rentabilidad no podría suponer una finalidad legítima suficientemente fuerte como para justificar un trato discriminatorio, sí que lo podría ser la continuidad de la empresa, en el sentido de que la no aplicación de la medida diferenciadora implicara la falta de viabilidad económica de la empresa. 
Dicho esto, conviene detenernos un momento para volver a analizar el uso que le dan las empresas a estos sistemas de evaluación. Como hemos visto, la primera finalidad de estos sistemas es que los clientes puedan dar su opinión acerca de la prestación de servicios para que la empresa pueda adaptar la prestación de servicios a sus necesidades y para generar confianza en sus clientes. Sin embargo, otra funcionalidad que tienen estos sistemas es la de sustituir las tareas de dirección, evaluación y compensación, que son de las funciones de gestión más importantes de los mandos intermedios49. Para visualizarlo podríamos decir que hay una función externa que consiste en conocer las opiniones de los clientes y otra función interna que consiste en gestionar esas evaluaciones y transformarlas en un control y compensación de los resultados del trabajo.

Como se indica en la citada Sentencia 53/2019 del Juzgado de lo Social no33 de Madrid: "[e]n el siglo XX la evaluación se realizaba directamente por las personas que tenían encomendada esa misión dentro de la estructura empresarial y se atendía esencialmente a la presencia en el centro de trabajo en el horario preestablecido. La contraprestación era el salario a cambio del tiempo empleado en trabajar. Con las TIC se da un notable impulso a otras formas de evaluación y retribución. No es preciso el control directo por otra persona, el mando intermedio, ya que esta tarea puede encomendarse a sistemas de control automatizados sean cámaras, ordenadores, sistemas de geolocalización, etc.".

En este plano, el sistema evaluativo actúa como una verdadera herramienta de management que permite a la empresa gestionar a una gran cantidad de trabajadores altamente dispersados sin emplear a un solo trabajador en las tareas de dirección, evaluación y compensación.50 Es cierto que la empresa no se desentiende completamente de estas funciones, pero simplemente se dedica a emitir unas directrices que suelen estar, a su vez, automatizadas. El ejemplo de Uber nos permite ver como la aplicación envía automáticamente a los trabajadores mensajes con las quejas más frecuentes de los clientes en la zona donde presta sus servicios el trabajador51. En este sentido, la aplicación realiza las funciones del jefe de personal y, mediante el sistema de evaluación, la empresa se ahorra una gran cantidad de trabajadores y de costes.

En relación con lo anterior, entendemos que la empresa podría argumentar en este sentido que la justificación objetiva y razonable del sistema de evaluación es, más allá del control de la prestación de servicios para satisfacer el cliente, la necesidad de

49 Ducato, Kullman And RocA, op. cit., p. 2.

50 Ibídem

51 Rosenblat, Levy, Barocas AND HwANG, op. cit., p. 4. 
gestionar el rendimiento de los trabajadores mediante este sistema para mantener la competitividad de la empresa y fomentar la calidad y productividad de los trabajadores.

A modo de conclusión, hemos analizado la jurisprudencia actual en materia de discriminación por parte de las exigencias de los clientes y hemos visto que, en el presente caso, se podría dar una discriminación indirecta por razón de sexo o de raza, atendiendo a los estudios que se han analizado en la primera parte del artículo. No obstante, cabe valorar la posibilidad de que exista una justificación objetiva y razonable en cuanto a la necesidad de la empresa de mantener la competitividad de la empresa y fomentar la calidad y productividad de los trabajadores, vinculando el sistema de evaluación a la viabilidad económica de la empresa y al principio de libertad de empresa. Esta vinculación se debe a las posibilidades que ofrecen estos sistemas en cuanto a la capacidad de conocer las exigencias de los clientes, pero sobre todo por las ventajas que ofrecen en materia de gestión de los resultados, compensación y fomento de la productividad de los trabajadores.52

Sin embargo, aún si se entendiera que existe legalmente un caso de discriminación, la demanda tendría una serie de complicaciones que vamos a tratar en este próximo apartado.

\section{2. ¿Sería jurídicamente viable una reclamación por discriminación?}

En este artículo hemos querido tener en cuenta, no solamente si existe discriminación o no en la utilización de estos sistemas de evaluación, sino también si podría prosperar una reclamación de este tipo, ya que solamente en ese caso se estaría protegiendo de forma efectiva los derechos de los trabajadores. Dada la extensión que podría tener el análisis profundo de esta cuestión, nos limitaremos a apuntar alguna de las opciones que existirían para tramitar una demanda por discriminación del sistema de evaluación.

En este sentido, debemos recordar que en las demandas de discriminación se produce la inversión de la carga de la prueba si el trabajador presenta indicios suficientes de discriminación, tal y como prevé el artículo 96 de la Ley Reguladora de la Jurisdicción Social (LRJS). Para presentar los indicios de discriminación, sería conveniente que el trabajador tuviera acceso a los datos concretos del sistema de evaluación de la empresa y, por lo tanto, a las evaluaciones de los demás trabajadores o a las diferencias salariales correspondientes.

Para solicitar esa información, el trabajador podría acudir al artículo 76 LRJS que regula la solicitud de actos preparatorios y diligencias preliminares donde se establece la 52 DUCATO, KUllman AND Roca, op. cit., p. 2. 
posibilidad de solicitar al órgano judicial que el demandado aporte algún documento cuyo conocimiento sea necesario para elaborar la demanda. Otra alternativa es pedir al Comité de la Empresa que solicite esa información a la empresa, en virtud del artículo 64 o incluso del artículo 28.2 del Estatuto de los Trabajadores. Este último artículo exige al empresario llevar un registro salarial distribuido por grupos profesionales, categorías profesionales o puestos de trabajo de igual valor, a los efectos de detectar brechas de género en las empresas.

Dicho registro, junto con el plan de igualdad previsto en el artículo 45 de la Ley Orgánica 3/2007, de 22 de marzo para la igualdad efectiva de mujeres y hombres podría facilitar la detección de situaciones discriminatorias derivadas de sistemas como los analizados en el presente artículo y, en consecuencia, podrían facilitar una demanda en este sentido.

En cuanto a las evaluaciones de los demás trabajadores, es lógico pensar que éstas serían un dato de carácter personal y que, por lo tanto, estarían emparadas por la normativa de Protección de Datos. Pues bien, en principio parece que nada impediría a la empresa transmitir los datos de forma agregada y despersonalizada, de forma que entendemos que, en el caso de que el demandante recibiera dichos datos, no se estaría vulnerando el derecho a la protección de datos personales. Además, el trabajador debería solicitar a la empresa que filtrara los datos teniendo en cuenta la causa discriminatoria que se alega.

Finalmente, es importante remarcar que la información que obtendría el trabajador perjudicado sería información estadística, que como ya hemos mencionado anteriormente, resulta muy relevante a la hora de probar si existe o no existe discriminación indirecta53. En consecuencia, si el trabajador llegara a poder tener los datos agregados de las retribuciones de los trabajadores, el análisis de si la medida perjudica o no perjudica a un grupo de trabajadores por las causas discriminatorias previstas en la ley no distaría mucho del análisis realizado en cualquier otro tipo de demanda por discriminación indirecta.

\section{Posibles modificaciones del sistema de evaluación}

A continuación, apuntaremos brevemente algunos de los cambios que se podrían realizar en los sistemas de evaluación para evitar que se produjera una situación discriminatoria como resultado de la aplicación de un sistema de evaluación como el descrito.

53 STC 253/2004 de 22 de diciembre (núm. rec. 2045/1998). 
En primer lugar, como ya hemos expuesto anteriormente, una de las medidas fundamentales para evitar que estos sistemas incurrieran en una discriminación efectiva sería que la propia empresa desarrollara procesos de control internos y analizara si en el sistema de evaluación de su empresa se produce algún tipo de discriminación. En este sentido, tenemos como ejemplo el estudio realizado sobre la existencia de discriminación por razón de sexo en las evaluaciones de los comerciales por parte de los clientes de WordStream. Ese estudio se elaboró a iniciativa de la propia empresa, para analizar si la discriminación también afectaba a la empresa.

Este análisis del resultado del sistema permitiría a la empresa poder compensar aquellas diferencias valorativas causadas por los sesgos discriminatorios. Por ejemplo, la empresa podría calcular la diferencia global en las valoraciones de hombres y mujeres y obtener el resultado que, de media, los trabajadores tienen 20 puntos más de valoración que las mujeres. Para compensar esa situación, la empresa podría sumar 20 puntos a cada una de las trabajadoras, de modo que se mantendría el sistema para las diferencias individuales, pero no se tendría en cuenta el efecto que tiene la diferencia de sexo.

Otra posible mejora sería limitar al máximo las variables de evaluación que sean más subjetivas y sustituirlas por variables lo más objetivas posible. Por ejemplo, sustituir la simple valoración de una a cinco estrellas por preguntas concretas de la prestación de servicios del trabajador. A modo de ejemplo, la valoración de Airbnb consiste en una valoración global del huésped y su habitación, pero también tiene una parte objetiva sobre hechos objetivos como si el huésped les había proporcionado toallas y sábanas54.

Asimismo, también sería interesante que el trabajador tuviera el derecho a conocer quién ha sido el cliente que le ha puesto una valoración en concreto y, si está en desacuerdo con ella, poder ofrecer una réplica a dicha evaluación. De esa forma, la empresa tendría la posibilidad de eliminar valoraciones que hayan sido claramente discriminatorias.

Finalmente, existe una corriente55 que aboga por la regulación de éste tipo de sistemas mediante negociación colectiva, con la intención de que los trabajadores tengan cierto control sobre la forma en la que serán evaluados y tengan conocimiento de los algoritmos a los que se somete su evaluación. Esta opción pretende reducir el impacto negativo que las nuevas tecnologías puedan tener en la calidad del trabajo y, además, puede suponer un instrumento de mejora de la fiabilidad de los sistemas de evaluación.

54 AIRBNB, op. cit.

55 De Stefano, V. 'Negotiating the Algorithm': Automation, Artificial Intelligence and Labour Protection (May 16, 2018). Comparative Labor Law \& Policy Journal, Forthcoming, p. 21. 


\section{Conclusión}

Como conclusión, de los estudios analizados podemos afirmar que estos sistemas de evaluación constituyen un método innovador de obtener una gran cantidad de información sobre las preferencias de los clientes. Además, estos sistemas permiten el establecimiento de un contexto de confianza entre el cliente y el prestador del servicio.

A su vez, estos sistemas tienen una funcionalidad interna como herramienta para gestionar los resultados del trabajo de manera que permiten compensar a los trabajadores de manera automática teniendo en cuenta las evaluaciones que los clientes han hecho sobre ellos.

No obstante, el uso de estos sistemas para la evaluación de trabajadores está sujeto a posibles sesgos por parte de los clientes que pueden vincularse a causas discriminatorias. Además, como hemos apuntado, existen otras problemáticas referidas a la protección de datos de carácter personal y a la indefensión producida por dichos sistemas de evaluación.

Por lo tanto, vemos que la novedosa aparición de este tipo de sistemas plantea muchas cuestiones jurídicas en cuanto a su funcionamiento. Una de ellas es la discriminación indirecta que, como hemos argumentado, se podría producir por la implementación de un sistema de evaluación del trabajador por parte de los clientes.

Actualmente, el uso de estos sistemas es limitado por lo que el impacto que pueden tener en la sociedad es relativamente bajo. Sin embargo, como se ha expuesto, su uso en nuevos sectores de la mano de empresas como Uber que pretenden aplicar el mismo modelo de negocio en distintos ámbitos y la posible implementación de estos sistemas en sectores tradicionales, puede causar un incremento en la utilización de estos sistemas $y$, en consecuencia, un incremento del impacto de la posible discriminación que comportan.

En este sentido, creemos que son muy interesantes los estudios doctrinales que analizan los problemas que presentan las nuevas tecnologías en el ámbito del derecho laboral y que, además, plantean posibles soluciones a ellas. A falta de precedentes y jurisprudencia, creemos que es muy importante que la doctrina jurídica realice un ejercicio de previsión respecto de los conflictos que pueden producirse en el uso de estas nuevas tecnologías, para reducir el desfalque temporal que suele producirse entre los cambios tecnológicos y su regulación. 
Finalmente, esperemos que el presente artículo haya cumplido su función de acercar al lector la problemática surgida de la aplicación de sistemas de evaluación de los trabajadores basados en las opiniones de los clientes y que, a pesar de la falta de jurisprudencia específica, el artículo haya servido para despertar la curiosidad respecto de una cuestión que aún no ha sido ampliamente analizada en el ámbito del Derecho Laboral, pero que puede ser de gran relevancia en los próximos años.

\section{Bibliografía}

GARCÍA SALAS, A. I "Necesidades empresariales y derechos fundamentales de los trabajadores", Thomson Reuters, 2016.

AYRES, B AND JOLLS, "Race effects on eBay." The RAND Journal of Economics 46, no. 4, 2015, p. 891- 917.

AYRES ET AL. "To Insure Prejudice: Racial Disparities in Taxicab Tipping", 114 Yale L.J. 1613, 1616, 2005.

BARTLETT Y GULATI, “Discrimination by Costumers” Iowa Law Review, Vol. 102:223, 2013, p. 224-257.

BAROCAS y SELBST, "Big Data's Disparate Impact", 104 Calif. L. Rev. 671, 2016, p. 1382.

De STEFAnO, V. 'Negotiating the Algorithm': Automation, Artificial Intelligence and Labour Protection, Comparative Labor Law \& Policy Journal, 2018, p. 21.

DUCATO, KULLMAN Y ROCA, "Customer Ratings as a Vector for Discrimination in Employment Relations Pathways and Pitfalls for Legal Remedies". Proceedings of the Marco Biagi Conference, 2018 (January 31).

DOLEAC AND STEIN, "The visible hand: Race and online market outcomes." The Economic Journal 123, no. 572, 2013, p. 469-492

Edelman AND LuCA, "Digital Discrimination: The Case of Airbnb.com", 9 Harvard Bus. Sch. Negotiation, Orgs. \& Mkts. Unit, Working Paper No. 14-054, 2014.

EDELMAN, LUCA AND SVIRSKY "Racial Discrimination in the Sharing Economy: Evidence from a Field Experiment." American Economic Journal: Applied Economics, Forthcoming, 2016. 
GABRIEla GUTIÉRREZ ET AL., "The intersections of Race and Class and Woman in Academia" Utah State University Press, 2012.

Kahneman, D. “Thinking, fast and slow”. New York: Farrar, Straus and Giroux. 2011.

BALLESTER PASTOR, M.A. "Retos y Perspectivas de la discriminación laboral por razón de género”, Tirant Lo Blanch, 2016.

Mittelstad et AL., "The ethics of algorithms: Mapping the debate", 3 Big Data \& Society 1, 2016, p. 1.

PARRET, "Customer Discrimination in Restaurants: Dining Frequency Matters", 32, $J$. Lab. Res. 87, 2011, 100-03.

PASQUALE, The Black Box Society, Harvard University Press, 2015.

ROSENBLAT, LEVY, BAROCAS AND HWANG, "Discriminating Tastes: Uber's Customer Ratings as Vehicles for Workplace Discrimination", 9 Policy and Internet 3, 2016, p. 256-279,

Wang, X, Y GILHEANY, "The Model Minority? Not on Airbnb.com: A Hedonic Pricing Model to Quantify Racial Bias against Asian Americans.", Technology Science, September 1, 2015. 